\title{
Connectivity in Language Areas of the Brain in Cochlear Implant Users as Revealed by fNIRS
}

\author{
Colette M. McKay, Adnan Shah, Abd-Krim Seghouane, Xin Zhou, \\ William Cross and Ruth Litovsky
}

\begin{abstract}
Many studies, using a variety of imaging techniques, have shown that deafness induces functional plasticity in the brain of adults with late-onset deafness, and in children changes the way the auditory brain develops. Cross modal plasticity refers to evidence that stimuli of one modality (e.g. vision) activate neural regions devoted to a different modality (e.g. hearing) that are not normally activated by those stimuli. Other studies have shown that multimodal brain networks (such as those involved in language comprehension, and the default mode network) are altered by deafness, as evidenced by changes in patterns of activation or connectivity within the networks. In this paper, we summarise what is already known about brain plasticity due to deafness and propose that functional near-infra-red spectroscopy (fNIRS) is an imaging method that has potential to provide prognostic and diagnostic information for cochlear implant users. Currently, patient history factors account for only $10 \%$ of the variation in post-implantation speech understanding, and very few post-implantation behavioural measures of hearing ability correlate with speech understanding. As a non-invasive, inexpensive and user-friendly imaging method, fNIRS provides an opportunity to study both pre- and post-implantation brain function. Here, we explain the principle of fNIRS measurements and illustrate its use in studying brain network connectivity and function with example data.
\end{abstract}

C. M. McKay $(\triangle) \cdot$ A. Shah $\cdot$ X. Zhou $\cdot$ W. Cross

The Bionics Institute of Australia, Melbourne, Australia

e-mail: cmckay@bionicsinstitute.org

C. M. McKay $\cdot$ X. Zhou

Department of Medical Bionics, The University of Melbourne, Melbourne, Australia

A. Shah · A.-K. Seghouane

Department of Electrical and Electronic Engineering, The University of Melbourne, Melbourne, Australia

W. Cross

Department of Medicine, The University of Melbourne, Melbourne, Australia

R. Litovsky

Waisman Center, The University of Wisconsin-Madison, Madison, USA

(C) The Author(s) 2016

P. van Dijk et al. (eds.), Physiology, Psychoacoustics and Cognition in Normal and Impaired Hearing, Advances in Experimental Medicine and Biology 894,

DOI 10.1007/978-3-319-25474-6 34 
Keywords fNIRS $\cdot$ Cochlear implants $\cdot$ Deafness $\cdot$ Brain plasticity $\cdot$ Connectivity in brain networks

\section{Introduction}

\subsection{Deafness, Language and Brain Plasticity: Evidence from Imaging Studies}

Speech understanding involves complex multimodal networks involving vision, hearing and sensory motor areas as well as memory and frontal lobe functions mostly in the left hemisphere (LH), and encompasses a range of elements such as phonology, semantics, and syntactics. The right hemisphere (RH) has fewer specialised functions for language processing, and its role is mostly in the evaluation of the communication context (Vigneau et al. 2011). Imaging studies have shown that adults who have undergone periods of profound post-lingual deafness demonstrate changes in brain activity and function in language-associated brain areas that are not observed in normally-hearing individuals, and that further functional plasticity occurs as a result of cochlear implantation.

Lee et al. (2003) used positron emission tomography (PET) to compare restingstate activity in 9 profoundly deaf individuals and 9 age-matched normal-hearing controls. They found that glucose metabolism in some auditory areas was lower than in normally-hearing people, but significantly increased with duration of deafness, and concluded that plasticity occurs in the sensory-deprived mature brain. Later, they showed that children with good speech understanding 3 years after implantation had enhanced metabolic activity in the left prefrontal cortex and decreased metabolic activity in right Heschl's gyrus and in the posterior superior temporal sulcus before implantation compared to those with poor speech understanding (Lee et al. 2007). They argued that increased activity in the resting state in auditory areas may reflect cross modal plasticity that is detrimental to later success with the cochlear implant (CI). Recently, Dewey and Hartley (2015) used functional near infrared spectroscopy (fNIRS) to demonstrate that the auditory cortex of deaf individuals is abnormally activated by simple visual stimuli.

Not all studies have suggested detrimental effects of brain changes due to deafness on the ability to adapt to listening with a CI. Two studies by Giraud et al used PET to study the activity induced by speech stimuli in CI users. The first showed that, compared to normal-hearing listeners, they had altered functional specificity of the superior temporal cortex, and exhibited contribution of visual regions to sound recognition (Giraud et al. 2001a). Secondly, the contribution of the visual cortex to speech recognition increased over time after implantation (Giraud et al. 2001b), suggesting that the CI users were actively using enhanced audio-visual integration to facilitate their learning of the novel speech sounds received through the CI. In contrast, Rouger et al. (2012) suggested a negative impact of cross modal plastic- 
ity: they found that the right temporal voice area (TVA) was abnormally activated in CI users by a visual speech-reading task and that this activity declined over time after implantation while the activity in Broca's area (normally activated by speech reading) increased over time after implantation. Coez et al. (2008) also used PET to study activation by voice stimuli of the TVA in CI users with poor and good speech understanding. The voice stimuli induced bilateral activation of the TVA along the superior temporal sulcus in both normal hearing listeners and CI users with good speech understanding, but not in CI users with poor understanding. This result is consistent with the proposal of Rouger et al that the TVA is 'taken over' by visual speech reading tasks in CI users who do not understand speech well. Strelnikov et al. (2013) measured PET resting state activity and activations elicited by auditory and audio-visual speech in CI users soon after implantation and found that good speech understanding after 6 months of implant use was predicted by a higher activation level of the right occipital cortex and a lower activation in the right middle superior temporal gyrus. They suggested that the pre-implantation functional changes due to reliance on lip-reading were advantageous to development of good speech understanding through a CI via enhanced audio-visual integration. In summary, functional changes that occur during deafness can both enhance and degrade the ability to adapt to CI listening, perhaps depending on the communication strategy each person used while deaf.

Lazard et al. have published a series of studies using functional magnetic resonance imaging (fMRI), in which they related pre-implant data with post-implant speech understanding. They found that, when doing a rhyming task with written words, CI users with later good speech understanding showed an activation pattern that was consistent with them using the normal phonological pathway to do the task, whereas those with poor outcomes used a pathway normally associated with lexical-semantic understanding (Lazard et al. 2010). They further compared activity evoked by speech and non-speech imageries in the right and left posterior superior temporal gyrus/supramarginal gyrus (PSTG/SMG) (Lazard et al. 2013). These areas are normally specialised for phonological processing in the left hemisphere and environmental sound processing in the right hemisphere. Their results suggested the abnormal recruitment of the right PSTG/SMG region for phonological processing.

In summary, studies have shown functional changes due to periods of deafness, some of which are detrimental and some advantageous to post-implant speech understanding. It is evident that the functional changes involve not just the auditory cortex, but the distributed multimodal language networks and that reliance on lipreading while deaf may be a major factor that drives functional changes.

\subsection{Functional Near Infra-red Spectroscopy (fNIRS)}

Near infra-red (NIR) light (wavelengths $650-1000 \mathrm{~nm}$ ) is relatively transparent to human tissues. The absorption spectra of oxygenated and de-oxygenated haemoglobin ( $\mathrm{HbO}$ and $\mathrm{HbR}$ respectively) for NIR light differ in that HbO maximally ab- 
sorbs light of longer wavelength (900-1000 nm) whereas HbR maximally absorbs light of shorter wavelength. These differential absorption spectra make it possible to separate out changes in the concentration of $\mathrm{HbO}$ and $\mathrm{HbR}$. In response to neural activity in the brain, an increase in oxygenated blood is directed to the region, resulting in a drop in de-oxygenated blood. Thus $\mathrm{HbO}$ and $\mathrm{HbR}$ concentrations change in opposite directions in response to neural activity (Shah and Seghouane 2014). Changes in $\mathrm{HbO}$ and $\mathrm{HbR}$ resulting from a neural response to a stimulus or due to resting state activity can be analysed to produce activation patterns and also to derive connectivity measures between regions of interest.

In the fNIRS imaging system optodes are placed at various locations on the scalp. Each fNIRS channel consists of a paired source and detector. The source emits a light beam, directed perpendicular to the scalp surface, and the detector detects the light emerging from the brain. The detected light beam has scattered in a banana-shaped pathway through the brain reaching a depth of approximately half the distance between the source and detector. In a montage of multiple optodes, each source can be associated with a number of surrounding detectors to form a multi-channel measurement system. In continuous wave systems, two frequencies of light are emitted by the diodes, and the signals at each source are frequency-modulated at different rates to facilitate separation of the light from different sources at the same detector position.

For studying language areas in the brain in CI users, fNIRS offers some advantages over other imaging methods: compared to PET it is non-invasive; in contrast to fMRI it can be used easily with implanted devices, is silent, and is more robust to head movements; compared to EEG/MEG it has much greater spatial resolution, and in CI users is free from electrical or magnetic artifacts from the device or the stimuli. The portability, low-cost, and patient-friendly nature of fNIRS (similar to EEG) makes it a plausible method to contribute to routine clinical management of patients, including infants and children.

However, there are also limitations of fNIRS. The spatial resolution is limited by the density of the optodes used, and is not generally as good as that found with fMRI or PET. The depth of imaging is limited, so that it is only suitable for imaging areas of the cortex near the surface [although other designs of fNIRS systems that use lasers instead of diodes and use pulsatile stimuli can derive 3-dimensional images of the brain, at least in infants (Cooper et al. 2014)]. However, fNIRS has been successfully used in a range of studies to determine effects on language processing in hearing populations of adults and children (Quaresima et al. 2012), therefore it shows promise for assessing language processing in deaf populations and those with cochlear implants.

In this paper we describe methods and present preliminary data for two investigations using fNIRS to compare CI users and normally-hearing listeners. In the first experiment, we measure resting state connectivity, and in the second experiment we compare the activation of cortical language pathways by visual and auditory speech stimuli. 


\section{Methods}

\section{1 fNIRS Equipment and Data Acquisition}

Data were acquired using a multichannel 32 optode (16 sources and 16 detectors) NIRScout system. Each source LED emitted light of two wavelengths (760 and $850 \mathrm{~nm}$ ). The sources and detectors were mounted in a pre-selected montage using an EASYCAP with grommets to hold the fNIRS optodes, which allowed registration of channel positions on a standard brain template using the international 10-20 system. To ensure optimal signal detection in each channel, the cap was fitted first, and the hair under each grommet moved aside before placing the optodes in the grommets. For CI users, the cap was fitted over the transmission coil with the speech processor hanging below the cap. The data were exported into MatLab or nirsLAB for analysis.

\subsection{Resting-State Connectivity in CI Users Using fNIRS}

In this study we compare the resting state connectivity of a group of normal hearing listeners compared to a group of experienced adult CI users. Brain function in the resting state, or its default-mode activity (Raichle and Snyder 2007) is thought to reflect the ability of the brain to predict changes to the environment and track any deviation from the predicted. Resting state connectivity (as measured by the correlation of resting state activity between different cortical regions) is influenced by functional organisation of the brain, and hence is expected to reflect plastic changes such as those due to deafness. In this study, we hypothesised that CI users would exhibit resting-state connectivity that was different from that of normally-hearing listeners.

We used a $4 \times 4$ montage of 8 sources and detectors ( 24 channels) in each hemisphere (Fig. 1d), which covered the auditory and somatosensory regions of the brain. Sources and detectors were separated by $3 \mathrm{~cm}$. Data in each channel were pre-processed to remove 'glitches' due to head movements and 'good' channels were identified by a significant cross-correlation $(r>0.75)$ between the data for the two wavelengths. Any 'poor' channels were discarded. The data were then converted to $\mathrm{HbO}$ and $\mathrm{HbR}$ based on a modified Beer-Lambardt Law (Cope et al. 1988). Since resting state connectivity is based upon correlations between activity in relevant channels, it is important to carefully remove any aspects of the data (such as fluctuations from heartbeat, breathing, or from movement artifacts that are present in all channels) that would produce a correlation but is not related to neural activity. These processing steps to remove unwanted signals from the data included: minimizing regional drift using a discrete cosine transform; removing global drift using PCA 'denoising' techniques, low-pass filtering $(0.08 \mathrm{~Hz})$ to remove heart and breathing fluctuations. Finally the connectivity between channels was calculated using Pearson's correlation, r. To account for missing channels, the data were reduced to 9 regions of interest in each hemisphere by averaging the data from groups of 4 channels. 

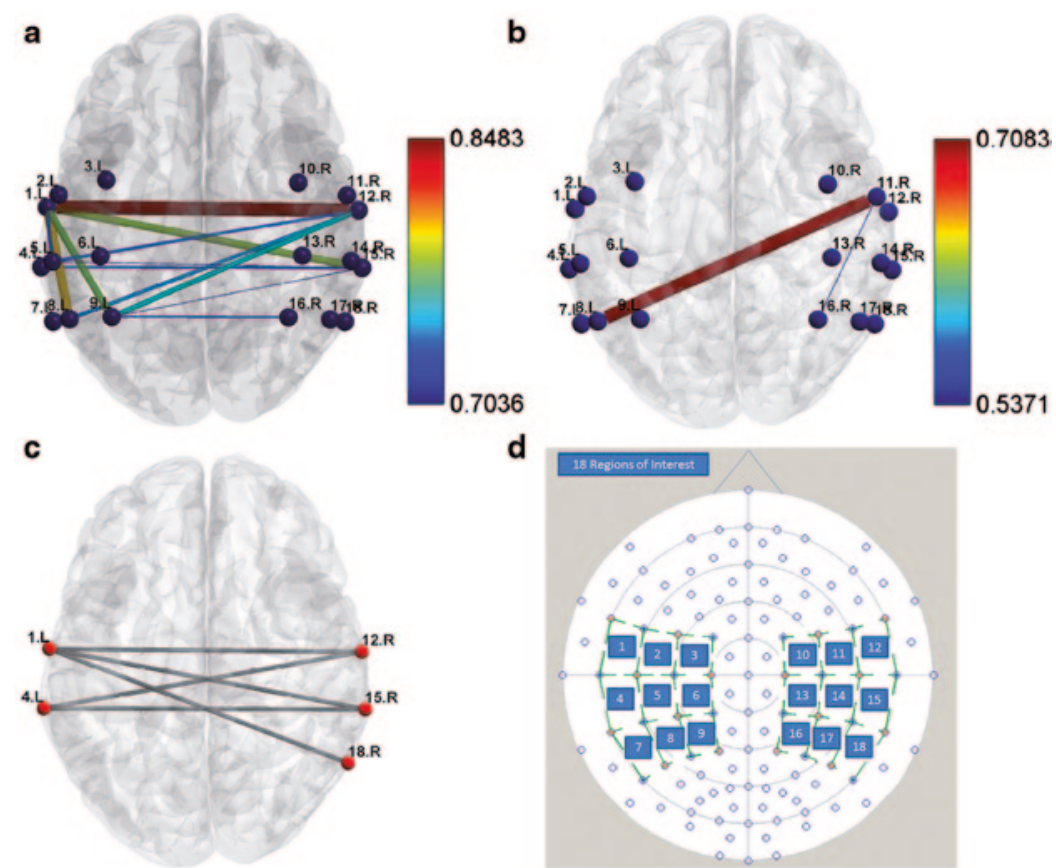

Fig. 1 Mean resting state connectivity in groups of 5 normal-hearing $(\mathrm{NH})$ listeners $(\mathbf{a})$ and $5 \mathrm{CI}$ users (b). The line colour and thickness denote the strength of connectivity (r) in the different brain regions of interest (colour bars denotes r-values). Panel $\mathbf{c}$ shows the channels that are significantly more highly connected in NH compared to CI listeners. Panel d shows the montage used and the 18 regions of interest

\subsection{Language Networks in CI Users Using fNIRS}

In the second study, we aimed to measure the activity in regions in the brain involved in language processing in response to auditory and visual speech stimuli. We hypothesised that, compared to normal-hearing listeners, CI users would show altered functional organisation, and furthermore that this difference would be correlated with lip-reading ability and with their auditory speech understanding.

The optode montage for this experiment is shown in Fig. 2a. fNIRS data were collected using a block design (12-s stimulus blocks separated by 15-25-s silent gaps and preceded by resting state baseline) in two sessions. In the first session, stimuli were visual and auditory words: in the second session stimuli were auditory and audiovisual sentences. The auditory stimuli were presented via EAR-4 insert ear phones in normally-hearing listeners, or via direct audio input for the CI users. Sounds were only presented to the right ear of subjects (the CI users were selected to have right-ear implants). 
a

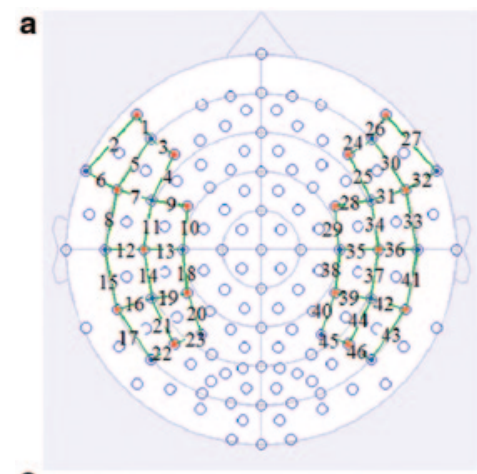

c

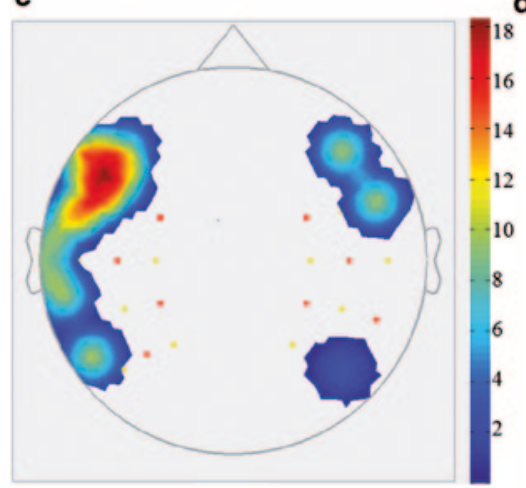

b
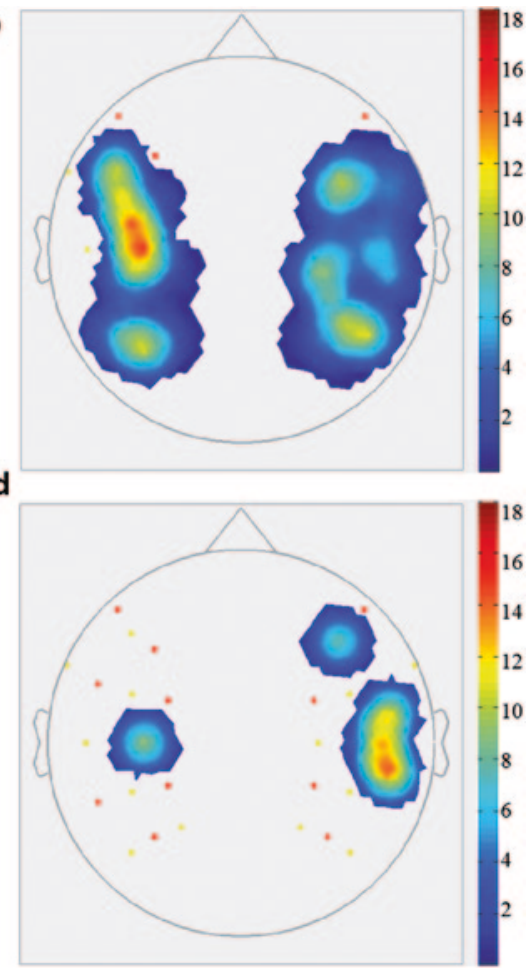

Fig. 2 Task-related activation due to audiovisual sentences (sound in right ear). a optode montage. b mean activation pattern from $10 \mathrm{NH}$ listeners. $\mathbf{c}$ and $\mathbf{d}$ activation patterns from individual CI users with good and poor speech understanding, respectively. The colour bar scale denotes the significance (t-value) of the activation compared to resting state

\section{Results}

\subsection{Preliminary fNIRS Data for Resting-State Connectivity}

Figure 1 shows data for mean resting state connectivity in groups of (A) 5 normalhearing (NH) listeners, and (B) 5 CI users: panel C shows channels that are more highly connected in the NH listeners than in the CI users $(p<0.001)$, using Network Based Statistics (Zalesky et al. 2010, pp. 1197-1207). All NH listeners tested to date have shown particularly strong connectivity between analogous regions in left and right hemispheres, consistent with other studies using fNIRS (Medvedev 2014). In contrast, the CI users in this group have consistently shown lower interhemispheric connectivity than the NH listeners. 


\subsection{Preliminary fNIRS Data for Language Networks}

Figure 2 illustrates the task-related activation pattern evoked by audiovisual sentences, comparing the mean pattern from a group of $10 \mathrm{NH}$ listeners to example patterns from two individual CI users with good and poor speech understanding. All NH listeners showed a similar activation pattern in wide language-associated regions in both hemispheres with asymmetry favouring the left hemisphere. The CI user with good speech understanding (100\% correct sentences in quiet) shows a generally similar pattern to that of the NH listeners, with left hemisphere dominance but greater activation in frontal areas (Broca's). In contrast, the CI user with poor speech understanding ( $<50 \%$ correct) has very little significant left-hemisphere activity, and little activation out of primary and associated auditory areas. Further work is being undertaken to compare activation patterns and connectivity in language pathways between $\mathrm{CI}$ users and NH listeners, and to correlate relevant differences to individual behavioural measures of speech understanding and lip-reading ability.

\section{Discussion}

Our preliminary data show that fNIRS has the potential to provide insight into the way that brain functional organisation is altered by deafness and subsequent cochlear implantation. The fNIRS tool may be particularly useful for longitudinal studies that track changes over time, so that the changes in brain function can be correlated with simultaneous changes in behavioural performance. In this way, new knowledge about functional organisation and how it relates to an individual's ability to process language can be gained. For example, an fNIRS pre-implant test may, in the future, provide valuable prognostic information for clinicians and patients, and provide guidance for individual post-implant therapies designed to optimise outcomes. Routine clinical use of fNIRS is feasible due to its low-cost. It will be particularly useful in studying language development in deaf children due to its patient-friendly nature.

Acknowledgments This research was supported by a veski fellowship to CMM, an Australian Research Council Grant (FT130101394) to AKS, a Melbourne University PhD scholarship to ZX, the Australian Fulbright Commission for a fellowship to RL, the Lions Foundation, and the Melbourne Neuroscience Institute. The Bionics Institute acknowledges the support it receives from the Victorian Government through its Operational Infrastructure Support Program.

Open Access This chapter is distributed under the terms of the Creative Commons AttributionNoncommercial 2.5 License (http://creativecommons.org/licenses/by-nc/2.5/) which permits any noncommercial use, distribution, and reproduction in any medium, provided the original author(s) and source are credited.

The images or other third party material in this chapter are included in the work's Creative Commons license, unless indicated otherwise in the credit line; if such material is not included in the work's Creative Commons license and the respective action is not permitted by statutory regulation, users will need to obtain permission from the license holder to duplicate, adapt or reproduce the material. 


\section{References}

Coez A, Zilbovicius M, Ferrary E, Bouccara D, Mosnier I, Ambert-Dahan E, Bizaguet E, Syrota A, Samson Y, Sterkers O (2008) Cochlear implant benefits in deafness rehabilitation: PET study of temporal voice activations. J Nucl Med 49(1):60-67. doi:10.2967/jnumed.107.044545

Cooper RJ, Magee E, Everdell N, Magazov S, Varela M, Airantzis D, Gibson AP, Hebden JC (2014) MONSTIR II: a 32-channel, multispectral, time-resolved optical tomography system for neonatal brain imaging. Rev Sci Instrum 85(5):053105. doi:10.1063/1.4875593

Cope M, Delpy DT, Reynolds EO, Wray S, Wyatt J, van der Zee P (1988) Methods of quantitating cerebral near infrared spectroscopy data. Adv Exp Med Biol 222:183-189

Dewey RS, Hartley DE (2015) Cortical cross-modal plasticity following deafness measured using functional near-infrared spectroscopy. Hear Res. doi:10.1016/j.heares.2015.03.007

Giraud AL, Price CJ, Graham JM, Frackowiak RS (2001a) Functional plasticity of language-related brain areas after cochlear implantation. Brain 124(Pt 7):1307-1316

Giraud AL, Price CJ, Graham JM, Truy E, Frackowiak RS (2001b) Cross-modal plasticity underpins language recovery after cochlear implantation. Neuron 30(3):657-663

Lazard DS, Lee HJ, Gaebler M, Kell CA, Truy E, Giraud AL (2010) Phonological processing in post-lingual deafness and cochlear implant outcome. Neuroimage 49(4):3443-3451. doi:10.1016/j.neuroimage.2009.11.013

Lazard DS, Lee HJ, Truy E, Giraud AL (2013) Bilateral reorganization of posterior temporal cortices in post-lingual deafness and its relation to cochlear implant outcome. Hum Brain Mapp 34(5):1208-1219. doi:10.1002/hbm.21504

Lee JS, Lee DS, Oh SH, Kim CS, Kim JW, Hwang CH, Koo J, Kang E, Chung JK, Lee MC (2003) PET evidence of neuroplasticity in adult auditory cortex of postlingual deafness. J Nucl Med 44(9): 1435-1439

Lee HJ, Giraud AL, Kang E, Oh SH, Kang H, Kim CS, Lee DS (2007) Cortical activity at rest predicts cochlear implantation outcome. Cereb Cortex 17(4):909-917

Medvedev AV (2014) Does the resting state connectivity have hemispheric asymmetry? A near-infrared spectroscopy study. Neuroimage 85(Pt 1):400-407. doi:10.1016/j.neuroimage.2013.05.092

Quaresima V, Bisconti S, Ferrari M (2012) A brief review on the use of functional near-infrared spectroscopy (fNIRS) for language imaging studies in human newborns and adults. Brain Lang 121(2):79-89. doi:10.1016/j.bandl.2011.03.009

Raichle ME, Snyder AZ (2007) A default mode of brain function: a brief history of an evolving idea. Neuroimage 37(4):1083-1090; discussion 1097-1089. doi:10.1016/j.neuroimage.2007.02.041

Rouger J, Lagleyre S, Demonet JF, Fraysse B, Deguine O, Barone P (2012) Evolution of crossmodal reorganization of the voice area in cochlear-implanted deaf patients. Hum Brain Mapp 33(8):1929-1940. doi:10.1002/hbm.21331

Shah A, Seghouane AK (2014) An integrated framework for joint HRF and drift estimation and $\mathrm{HbO} / \mathrm{HbR}$ signal improvement in fNIRS data. IEEE Trans Med Imaging 33(11):2086-2097. doi:10.1109/TMI.2014.2331363

Strelnikov K, Rouger J, Demonet JF, Lagleyre S, Fraysse B, Deguine O, Barone P (2013) Visual activity predicts auditory recovery from deafness after adult cochlear implantation. Brain 136(Pt 12):3682-3695. doi:10.1093/brain/awt274

Vigneau M, Beaucousin V, Herve PY, Jobard G, Petit L, Crivello F, Mellet E, Zago L, Mazoyer B, Tzourio-Mazoyer N (2011) What is right-hemisphere contribution to phonological, lexico-semantic, and sentence processing? Insights from a meta-analysis. Neuroimage 54(1):577-593. doi:10.1016/j.neuroimage.2010.07.036

Zalesky A, Fornito A, Bullmore ET (2010) Network-based statistic: identifying differences in brain networks. Neuroimage 53(4):1197-1207 